\title{
KNOWLEDGE DISCOVERY
}

\section{Applying Knowledge \\ Discovery to Predict \\ Woter-Supply Consumption}

\section{Aijun An, Christine Chan, Ning Shan, Nick Cercone, and Wojciech Ziarko, University of Regina, Saskatchewan, Canada}

PTIMIZING CONTROL OF OPERations in a municipal water-distribution system can reduce electricity costs and realize other economic benefits. However, optimal control requires an ability to precisely predict short-term water demand so that minimumcost pumping schedules can be prepared. One of the objectives of our project to develop an intelligent system for monitoring and controlling municipal water-supply systems is to ensure optimal control and reduce energy costs. Hence, prediction of water demand is essential. In this article, we present an application of a rough-set approach for automated discovery of rules from a set of data samples for daily water-demand predictions. The database contains 306 training samples, covering information on seven environmental and sociological factors and their corresponding daily volume of distribution flow.

\section{The problem domain}

The problem domain is typical of a waterdistribution system of moderate-sized cities in North America. The sources of water are a lake and several underground wells. Water is first pumped to reservoirs at various locations in the city and then from the reservoirs to the distribution system, or to another reservoir when it is necessary to adjust water levels.

\author{
A ROUGH-SET METHOD GENERATES PREDICTION RULES \\ FROM OBSERVED DATA, USING STATISTICAL INFORMATION \\ INHERENT IN THE DATA TO HANDLE INCOMPLETE AND \\ AMBIGUOUS TRAINING SAMPLES. EXPERIMENTAL RESULTS \\ INDICATE THAT THIS METHOD PROVIDES MORE PRECISE \\ INFORMATION THAN IS AVAILABLE THROUGH KNOWLEDGE \\ ACQUISITION FROM HUMAN EXPERTS.
}

Pumps and valves, housed in pumping stations, control the system's pressures and flow rates. Human operators control the distribution system's operations at a central pumping station. The operators use heuristics or rules of thumb to minimize the cost of power used by pumps, to make demand forecasts, and to keep the water level of reservoirs within reasonable ranges. These heuristics depend on several economic, environmental, and sociological factors. A sample heuristic is,

If the weather in the last three days is hot and dry, and the weather in the next three days is expected to be hot and dry, and the time before high demand is expected to be less than or equal to eight hours, then use a large pump and run it for a short time.

Because several operators control the system, standardizing and optimizing the distri- bution system's operations is difficult. Documenting the heuristics of the most experienced operator in an intelligent system is one way to reduce operating costs in the supply and distribution of purified water.

To develop our expert system, we conducted knowledge acquisition through structured and unstructured interviews with human experts, and we obtained heuristics for costeffective water-utility operations. Analysis of these heuristics indicates that accurately estimating short-term (daily) forecasts of water demand is important so that energy-saving pumping schedules can be derived. However, the prediction of water demand is poorly understood-even by the experts, who approximate daily water demand based on their experience. The often inaccurate estimations result in inefficient operation of the water-dis- 
tribution system. The lack of knowledge about water-demand prediction translates to a gap in the expert system's knowledge base. In other words, manual knowledge acquisition by itself is inadequate for handling all the situations that can arise in a complex engineering application.

The relevant studies on demand prediction have focused on developing mathematical models based on statistical studies of historical data. ${ }^{1}$ In our project, we focused on using machine-learning techniques for generating rules on water-demand prediction, because mathematical modeling focuses on quantitative information alone. But in automated learning from observed data, both quantitative as well as qualitative variables are considered.

Because the database's training samples are incomplete and possibly ambiguous, exact decision rules cannot be derived by standard methods. ${ }^{2-5}$ We propose a method for generating classification rules from incomplete information. This method extends the roughset model, ${ }^{6}$ and uses statistical information to define the positive and negative regions of a concept. Each classification rule generated by our learning system is characterized by an uncertainty factor, which is the probability that an object matching the condition part of the rule belongs to the concept.

\section{Data collection and representation}

We obtained data on the water domain from the municipal water department in Regina, Saskatchewan, Canada, and Environment Canada. The former provided us with historical data on water consumption, and the latter on weather conditions.

Characteristics of water demand. The instantaneous consumption of water in an urban distribution system depends on the many industrial, commercial, public, and domestic consumers distributed throughout the area supplied. Factors such as weather conditions, seasonal variation, day of the week, and whether a particular day is an observed holiday can all influence this consumption. We have found that expert operators base their water-demand predictions primarily on weather-related considerations and day of the week. Therefore, in this article, we use weather conditions and day of the week as the condition attributes, and consumer water demand as the decision attribute.
Condition attributes. Seven factors can affect the daily consumption of water in a city (see Table 1). The first factor is day of the week, which we chose because of the observation that on weekends the daily totaldistribution flows are usually less than on weekdays. The city also has the power, through bylaws, to restrict watering of lawns on Wednesdays. Furthermore, Mondays are days of high water usage, because many people do their laundry on Mondays and because, in the summer, people water their lawns on the day after returning from a weekend at their cottages. The remaining factors are weather conditions: temperature, humidity, precipitation, and wind. We obtained the values of these factors from monthly meteorological summaries printed by Environment Canada.

Decision attribute. The total amount of water consumed by the city each day is calculated by summing the daily distribution flows metered at each pumping station of the city. The operator at the central control station records this number every day. The value varies from 50 megaliters in the coldest winter days to 180 megaliters in the summer.

Information systems. We assume that the given set of training samples represents the knowledge about the domain. In our approach, the training set is described by an information system. ${ }^{4}$ The objects in a universe $U$ are described by a set of attribute values.

Formally, an information system $S$ is a quadruple $\langle U, A, V, f\rangle$, where $U=\left\{x_{1}, x_{2}\right.$, $\left.\ldots, x_{N}\right\}$ is a finite set of objects, and $A$ is a finite set of attributes. The attributes in $A$ are further classified into two disjoint subsets: the condition attributes $C$ and the decision attribute $D$, such that $A=C \cup D$ and $C \cap D$ $=\emptyset \cdot V=\bigcup_{a \in A} V_{a}$ is a set of attribute values, where $V_{a}$ is the domain of attribute $a$ (the set of values of attribute $a$ ). $f: U \times A \rightarrow V$ is an information function that assigns particular values from domains of attributes to objects, such that $f\left(x_{i}, a\right) \in V_{a}$ for all $x_{i} \in U$ and $a \in A$.

The 306 training samples in our information system for water-demand prediction are objects that include daily information on the condition attributes and the decision attribute

\begin{tabular}{ll}
\hline Label & Condition attributes \\
\hline$a_{0}$ & Day of the week \\
$a_{1}$ & Maximum temperature \\
$a_{2}$ & Minimum temperature \\
$a_{3}$ & Average humidity \\
$a_{4}$ & Rainfall \\
$a_{5}$ & Snowfall \\
$a_{6}$ & Average wind speed \\
\hline
\end{tabular}

able 2. Information system for water-demand prediction.

\section{C}

$\begin{array}{lllll}a_{3} & a_{4} & a_{5} & a_{6} & D \\ 8 & 1 & 0 & 3 & 0 \\ 9 & 0 & 0 & 1 & 0 \\ 8 & 1 & 0 & 3 & 0 \\ 4 & 0 & 0 & 1 & 1 \\ 3 & 0 & 0 & 1 & 1 \\ 4 & 0 & 0 & 1 & 1 \\ 3 & 0 & 0 & 6 & 2 \\ 4 & 0 & 0 & 1 & 2\end{array}$

for 10 months, from March to December 1994. Table 2 lists eight of these objects. For the purpose of rough-set-based data analysis, we have generalized the information system by replacing the original attribute values with some discrete ranges - for example, we have discretized attribute $a_{2}$ (minimum temperature) into 10 categories, denoted as 0,1 , $2,3,4,5,6,7,8$, and 9 . For instance, categories 7 and 8 for $a_{2}$, which appear in Table 2 , stand for the ranges $(6.46,10.34]$ and (10.34, 14.22], respectively. (The unmatched brackets stand for a half-open and half-closed range. For example, 6.46 does not belong to the range $(6.46,10.34]$, but 10.34 does.)

The question of how to optimally discretize the attribute values is a subject of ongoing research; the method of discretization we have adopted is just one of many possibilities. Our experimental results are based on the whole set of training samples (the 306object information system).

\section{Data analysis}

In what follows, we present the basics of an extended rough-set model, referred to as the probabilistic model of rough sets. The probabilistic model underlies the rule-extraction technique adopted in our research.

Indiscernibility relation. An information system provides only partial information of the universe. That is, the objects described 
by the fixed set of selected attributes might be insufficient for characterizing the objects uniquely. Any two objects are indistinguishable from each other whenever they assume the same attribute values. This means we might not be able to distinguish all the objects solely by means of the admitted attributes and their values.

Given an information system $<U, A, V$, $f>$, let $B$ be a subset of $A$, and let $x_{i}$ and $x_{j}$ be members of $U$. A binary relation $R(B)$, called an indiscernibility relation, is defined as $R(B)=\left\{\left(x_{i}, x_{j}\right) \in U^{2} \mid \forall a \in B, f\left(x_{i}, a\right)=\right.$ $\left.f\left(x_{j}, a\right)\right\}$. We say that $x_{i}$ and $x_{j}$ are indiscernible to the set of attributes $B$ in $S$ if $f\left(x_{i}\right.$, $a)=f\left(x_{j}, a\right)$ for every $a \in B$. For example, in the information system shown in Table 2, $o b j_{1}$ and $o b j_{3}$ are indiscernible to the set of attributes $C \cup D$, and $o b j_{4}, o b j_{6}$, and $o b j_{8}$ are indiscernible to the set of condition attributes $C$.

$R(B)$ is an equivalence relation on $U$ for every $B \subseteq A$. Thus, we can define two natural equivalence relations, $R(C)$ and $R(D)$, on $U$ for an information system $S$. A concept $Y$ is an equivalence class of the relation $R(D)$. Without loss of generality, we can consider $D$ as a singleton set. Our objective is to construct decision rules for each concept. Given a concept $Y$, the partition of $U$ with respect to this concept is defined as $R^{*}(D)=\{Y, U-$ $Y\}=\{Y, \neg Y\}$.

Based on the set of condition attributes $C$, an object $x_{i}$ specifies the equivalence class $\left[x_{i}\right]_{R}$ of the relation $R(C)$ :

$$
\left[x_{i}\right]_{R}=\left\{x_{j} \in U \mid \forall a \in C, f\left(x_{j}, a\right)=f\left(x_{i}, a\right)\right\}
$$

We say that $x_{i} \in U$ definitely belongs to a concept $Y$ if $\left[x_{i}\right]_{R} \subseteq Y$, and that $x_{i} \in U$ possibly belongs to the concept $Y$ if $\left[x_{i}\right]_{R} \cap Y \neq \emptyset$.

We define conditional probabilities as

$$
\mathrm{P}\left(Y \mid\left[x_{i}\right]_{R}\right)=\frac{\mathrm{P}\left(Y \cap\left[x_{i}\right]_{R}\right)}{\mathrm{P}\left(\left[x_{i}\right]_{R}\right)}=\frac{\left|Y \cap\left[x_{i}\right]_{R}\right|}{\left.|| x_{i}\right]_{R} \mid}
$$

where $\mathrm{P}\left(Y \mid\left[x_{i}\right]_{R}\right)$ is the probability of occurrence of event $Y$ conditioned on event $\left[x_{i}\right]_{R}$. That is, $\mathrm{P}\left(Y \mid\left[x_{i}\right]_{R}\right)=1$ if and only if $\left[x_{i}\right]_{R} \subseteq Y ; \mathrm{P}\left(Y \mid\left[x_{i}\right]_{R}\right)>0$ if and only if $\left[x_{i}\right]_{R}$ $\cap Y \neq \emptyset$; and $\mathrm{P}\left(Y \mid\left[x_{i}\right]_{R}\right)=0$ if and only if $\left[x_{i}\right]_{R} \cap Y=\emptyset$.

Example 1. Let us consider the given information system in Table 2. The concepts in this information system, the equivalence classes on the relation $R(D)$, are

$$
\begin{aligned}
& Y_{0}=\left\{o b j_{1}, o b j_{2}, o b j_{3}\right\} \\
& Y_{1}=\left\{o b j_{4}, o b j_{5}, o b j_{6}\right\} \\
& Y_{2}=\left\{o b j_{7}, o b j_{8}\right\}
\end{aligned}
$$

The equivalence classes on the relation $R(C)$ are

$$
\begin{aligned}
X_{1} & =\left[o b j_{1}\right]_{R}=\left[o b j_{3}\right]_{R}=\left\{o b j_{1}, o b j_{3}\right\} \\
X_{2} & =\left[o b j_{2}\right]_{R}=\left\{o b j_{2}\right\} \\
X_{3} & =\left[o b j_{4}\right]_{R}=\left[o b j_{6}\right]_{R}=\left[o b j_{8}\right]_{R} \\
& =\left\{o b j_{4}, o b j_{6}, o b j_{8}\right\} \\
X_{4} & =\left[o b j_{5}\right]_{R}=\left\{o b j_{5}\right\} \\
X_{5} & =\left[o b j_{7}\right]_{R}=\left\{o b j_{7}\right\}
\end{aligned}
$$

Because $X_{4} \subseteq Y_{1}, o b j_{5}$ definitely belongs to concept $Y_{1}$. The objects $o b j_{4}, o b j_{6}$, and $o b j_{8}$ possibly belong to concept $Y_{1}$, because the intersection of their equivalence class, $X_{3}$, with $Y_{1}$ is not empty. Other objects do not belong to $Y_{1}$.

The conditional probability of each equivalence class is

$$
\begin{aligned}
& \mathrm{P}\left(Y_{1} \mid X_{1}\right)=0 \\
& \mathrm{P}\left(Y_{1} \mid X_{2}\right)=0 \\
& \mathrm{P}\left(Y_{1} \mid X_{3}\right)=2 / 3=0.667 \\
& \mathrm{P}\left(Y_{1} \mid X_{4}\right)=1 \\
& \mathrm{P}\left(Y_{1} \mid X_{5}\right)=0
\end{aligned}
$$

$\beta$-probabilistic approximation classification. Given an information system $S=\{U$, $A, V, f\}$ and an equivalence relation $R(C)$ (an indiscernibility relation) on $U$, an ordered pair $A S=\langle U, R(C)\rangle$ is called an approximation space $e^{4}$ based on the condition attributes $C$. The equivalence classes of the relation $R(C)$ are called elementary sets in $A S$, because they represent the smallest groups of objects that are distinguishable in terms of the attributes and their values. Let $Y \subseteq U$ be a subset of objects representing a concept, and $R^{*}(C)=\left\{X_{1}, X_{2}, \ldots, X_{n}\right\}=\left\{\left[x_{1}\right]_{R},\left[x_{2}\right]_{R}\right.$, $\left.\ldots,\left[x_{n}\right]_{R}\right\}$ be the collection of equivalence classes induced by the relation $R(C)$. In the standard rough-set model, the lower and upper approximations of a set $Y$ are defined by

$$
\underline{R(C)}(Y)=\bigcup_{\mathrm{P}\left(Y \mid X_{i}\right)=1}\left\{X_{i} \in R^{*}(C)\right\}
$$

and

$$
\overline{R(C)}(Y)=\bigcup_{\mathrm{P}\left(Y \mid X_{i}\right)>0}\left\{X_{i} \in R^{*}(C)\right\}
$$

The above definitions do not use the statistical information in the boundary region,

$$
\overline{R(C)}(Y)-R(C)(Y)
$$

For this reason, several extensions to the original rough-set model have been proposed. ${ }^{6-9,11}$ In our approach, we try to rectify this limitation by introducing a $\beta$-approximation space.

A $\beta$-approximation space $A S_{P}$ is a triple $\langle U, R(C), \mathrm{P}\rangle$, where $\mathrm{P}$ is a probability measure and $\beta$ is a real number in the range $(0.5$, $1]$. The $\beta$-approximation space $A S_{P}$ can be divided into the following regions:

$\beta$-positive region of the set $Y$ :

$$
\operatorname{POS}_{C}(Y)=\bigcup_{\mathrm{P}\left(Y \mid X_{i}\right) \geq \beta}\left\{X_{i} \in R^{*}(C)\right\}
$$

$\beta$-negative region of the set $Y$ :

$$
\mathrm{NEG}_{C}(Y)=\bigcup_{\mathrm{P}\left(Y \mid X_{i}\right)<\beta}\left\{X_{i} \in R^{*}(C)\right\}
$$

The $\beta$-positive region of the set $Y$ corresponds to all elementary sets of $U$ that can be classified into the concept $Y$ with conditional probability $\mathrm{P}\left(Y \mid X_{i}\right)$ greater than or equal to the parameter $\beta$. Similarly, the negative region of the set $Y$ corresponds to all elementary sets of $U$ that can be classified into the set $\neg Y$.

Let $x_{i} \in U$ be an object; $\operatorname{POS}_{C}(Y)$ and $\operatorname{NEG}_{C}(Y)$ are the positive and negative regions of the concept $Y$. The object $x_{i}$ is classified as belonging to the concept $Y$ if and only if $x_{i} \in \operatorname{POS}_{C}(Y)$, or to the complement $\neg Y$ of the concept $Y$ if and only if $x_{i} \in$ $\mathrm{NEG}_{C}(Y)$. We want to decide whether $x_{i}$ is in the concept $Y$ on the basis of the set of equivalence classes in $A S_{P}$ rather than on the basis of the set $Y$. This means we deal with $\operatorname{POS}_{C}(Y)$ and $\operatorname{NEG}_{C}(Y)$ instead of the set $Y$. If $x_{i} \in U$ is in $\operatorname{POS}_{C}(Y)$, it can be classified into the concept $Y$ with the conditional probability $\mathrm{P}\left(Y \mid X_{i}\right)$ greater than or equal to the parameter $\beta$.

Example 2. Following from Example 1, recall that $Y_{1}=\left\{o b j_{4}, o b j_{5}, o b j_{6}\right\}$. If we let $\beta$ $=1$, then the $\beta$-positive region of the set $Y_{1}$ is $\operatorname{POS}_{C}\left(Y_{1}\right)=\left\{o b j_{5}\right\}$, and the $\beta$-negative region of the set $Y_{1}$ is $\mathrm{NEG}_{C}\left(Y_{1}\right)=\left\{o b j_{1}, o b j_{2}, o b j_{3}\right.$, $\left.o b j_{4}, o b j_{6}, o b j_{7}, o b j_{8}\right\}$. If we let $\beta=0.6$, then the $\beta$-positive region of the set $Y_{1}$ is $\operatorname{POS}_{C}\left(Y_{1}\right)$ $=\left\{o b j_{4}, o b j_{5}, o b j_{6}, o b j_{8}\right\}$, and the $\beta$-negative region of the set $Y_{1}$ is $\operatorname{NEG}_{C}\left(Y_{1}\right)=\left\{o b j_{1}, o b j_{2}\right.$, $\left.o b j_{3}, o b j_{7}\right\}$.

Reduction of condition attributes. An information system often includes some condition attributes that do not provide any addi- 
Table 3. Reduct table with respect to $Y_{1}$ after reduction from Table 2.

tional information about the objects in $U$. Eliminating those attributes can reduce the complexity and cost of the decision process. We use the concept of a reduct in rough sets to describe the method of condition-attributes reduction.

Given an attribute-value system $S=<U, C$ $\cup\{d\}, V, f>$, an attribute $a$ is dispensable in $C$ with respect to $\{d\}$ if $\operatorname{POS}_{C-\{a\}}(Y)=$ $\operatorname{POS}_{C}(Y)$; otherwise $a$ is an indispensable attribute in $C$ with respect to $\{d\}$. A subset of condition attributes $B \subseteq C$ is a dependent set in $\mathrm{S}$ with respect to $\{d\}$ if a proper subset $K \subset B$ exists such that $\operatorname{POS}_{B}(Y)=\operatorname{POS}_{K}(Y)$; otherwise, $B$ is an independent set with respect to $\{d\}$. A reduct $C^{\prime}$ of attributes $C$ is a maximal independent subset of condition attributes with respect to $\{d\}{ }^{4}$

The procedure for finding a single reduct is very straightforward. Consider a condition attribute $a \in C$. If the $\beta$-positive region $\operatorname{POS}_{C-\{a\}}(Y)$ of the set $Y$ is the same as $\operatorname{POS}_{C}(Y)$, then the attribute $a$ is marked as being redundant and is removed from the set of condition attributes $C$. Other superfluous condition attributes can be removed in the same manner. The remaining set of condition attributes is a reduct. More than one reduct can exist for a given attribute-value system. Selection of a best reduct depends on the optimality criterion associated with the attributes. We can also assign significance values to attributes and base the selection on those values.

Example 3. The condition attributes $C$ of the information system in Table 2 has a total of seven reducts. Table 3 shows a reduced information system based on the reduct $\left\{a_{1}, a_{3}\right\}$ with respect to the concept $Y_{1}$. The objects with value 1 for attribute $Y_{1}$ belong to the $\beta$ positive region of the concept $Y_{1}$; the objects with value 0 for attribute $Y_{1}$ belong to the $\beta$ negative region of concept $Y_{1}$. Here, $\beta=0.6$.

\section{Generating decision rules}

Rule generation is a crucial task in any learning system. We now describe how decision rules are generated based on the reduct obtained in the previous section.

Probabilistic decision rules. Let $R^{*}$ (RED) $=\left\{X_{1}, X_{2}, \ldots, X_{n}\right\}$ be the collection of equivalence classes of the relation $R(\mathrm{RED})$, where RED is a reduct that is a reduced set of condition attributes $C$ in $S$, and let $R^{*}(D)=\{Y$,

\begin{tabular}{|c|c|c|c|c|c|}
\hline \multirow[b]{2}{*}{ OBJects } & \multicolumn{2}{|c|}{$C$} & \multirow[b]{2}{*}{$Y_{1}$} & \multirow{2}{*}{$\begin{array}{c}\text { NuMBER OF } \\
\text { OBJECTS IN } Y_{1}\end{array}$} & \multirow{2}{*}{$\begin{array}{c}\text { NUMBEER OF } \\
\text { OBJECTS IN } \neg Y_{1}\end{array}$} \\
\hline & $a_{1}$ & $a_{3}$ & & & \\
\hline$o b j_{4}, o b j_{6}, o b j_{8}$ & 6 & 4 & 1 & 2 & 1 \\
\hline$o b j_{5}$ & 5 & 3 & 1 & 1 & 0 \\
\hline$o b j_{1}, o b j_{3}$ & 6 & 8 & 0 & 0 & 2 \\
\hline$o b j_{2}$ & 5 & 9 & 0 & 0 & 1 \\
\hline$o b j_{7}$ & 6 & 3 & 0 & 0 & 1 \\
\hline
\end{tabular}

$\neg Y$ \} be the partition induced by the decision attribute. Each equivalence class $X_{i}$ of the equivalence relation $R(\mathrm{RED})$ is associated with a unique combination of values of attributes belonging to RED. This combination of values is called the description of the equivalence class $X_{i} \in R^{*}(\mathrm{RED})$. We can express the description of $X_{i}$ as

$$
\operatorname{Des}\left(X_{i}\right)=\bigwedge_{a \in \operatorname{RED}}\left(a=f\left(x_{i}, a\right)\right)
$$

where $\wedge$ denotes the conjunction operator, and $x_{i}$ is an object in the equivalence class $X_{i}$. Similarly, the descriptions of $Y$ and $\neg Y$ are

$$
\begin{aligned}
& \operatorname{Des}(Y)=\left(D=f\left(x_{i}, D\right)\right), \text { and } \\
& \operatorname{Des}(\neg Y)=\left(D \neq f\left(x_{i}, D\right)\right)
\end{aligned}
$$

where $D$ is the decision attribute and $x_{i} \in Y$.

The following decision rules describe the relationship between the partition $R^{*}(\mathrm{RED})$ and the partition $R^{*}(D)$ :

for $X_{i} \in R^{*}(\mathrm{RED})$,

$$
\begin{aligned}
& \operatorname{Des}\left(X_{i}\right) \rightarrow{ }^{C_{i}} \operatorname{Des}(Y), \text { if } \mathrm{P}\left(Y \mid X_{i}\right) \geq \beta \\
& \operatorname{Des}\left(X_{i}\right) \rightarrow{ }^{C_{i}} \operatorname{Des}(\neg Y) \text {, if } \mathrm{P}\left(Y \mid X_{i}\right)<\beta
\end{aligned}
$$

where $c_{i}$ is the uncertainty factor, which is equal to $\mathrm{P}\left(Y \mid X_{i}\right)$ in the first case and $1-\mathrm{P}(Y$ $\left.\mid X_{i}\right)$ in the second. This means that if an object $x_{i}$ satisfies the description $\operatorname{Des}\left(X_{i}\right)$ and if $\mathrm{P}\left(Y \mid X_{i}\right) \geq \beta$, then the object $x_{i}$ definitely belongs to $Y$ with uncertainty $c_{i}$. Similarly, if $\mathrm{P}\left(Y \mid X_{i}\right)<\beta$, then the object $x_{i}$ possibly belongs to the complementary concept $\neg Y$ with uncertainty $c_{i}$.

Example 4. Following from Example 3, let RED denote the reduct $\left\{a_{1}, a_{3}\right\}$. The collection $R^{*}(\mathrm{RED})$ of the equivalence classes of the relation $R(\mathrm{RED})$ is

$R^{*}(\mathrm{RED})=\left\{X_{1}, X_{2}, X_{3}, X_{4}, X_{5}\right\}=\left\{\left\{o b j_{4}\right.\right.$, $\left.o b j_{6}, o b j_{8}\right\},\left\{o b j_{5}\right\},\left\{o b j_{1}, o b j_{3}\right\},\left\{o b j_{2}\right\}$, $\left.\left\{o b j_{7}\right\}\right\}$
The descriptions of these equivalence classes are

$$
\begin{aligned}
& \operatorname{Des}\left(X_{1}\right)=\left(a_{1}=6\right) \wedge\left(a_{3}=4\right) \\
& \operatorname{Des}\left(X_{2}\right)=\left(a_{1}=5\right) \wedge\left(a_{3}=3\right) \\
& \operatorname{Des}\left(X_{3}\right)=\left(a_{1}=6\right) \wedge\left(a_{3}=8\right) \\
& \operatorname{Des}\left(X_{4}\right)=\left(a_{1}=5\right) \wedge\left(a_{3}=9\right) \\
& \operatorname{Des}\left(X_{5}\right)=\left(a_{1}=6\right) \wedge\left(a_{3}=3\right)
\end{aligned}
$$

The descriptions of $Y_{1}$ and $\neg Y_{1}$ are

$$
\begin{aligned}
& \operatorname{Des}\left(Y_{1}\right)=(D=1) \\
& \operatorname{Des}\left(\neg Y_{1}\right)=(D \neq 1)
\end{aligned}
$$

Because $Y_{1}=\left\{o b j_{4}, o b j_{5}, o b j_{6}\right\}$, we calculate the condition probabilities as

$$
\begin{aligned}
& \mathrm{P}\left(Y_{1} \mid X_{1}\right)=2 / 3=0.67 \\
& \mathrm{P}\left(Y_{1} \mid X_{2}\right)=1 \\
& \mathrm{P}\left(Y_{1} \mid X_{3}\right)=0 \\
& \mathrm{P}\left(Y_{1} \mid X_{4}\right)=0 \\
& \mathrm{P}\left(Y_{1} \mid X_{4}\right)=0
\end{aligned}
$$

We then obtain the decision rules with respect to the concept $Y_{1}$ as follows:

$$
\begin{array}{ll}
r_{1}^{+}: & \left(a_{1}=6\right) \wedge\left(a_{3}=4\right) \rightarrow^{0.67}(D=1) \\
r_{2}^{+}: & \left(a_{1}=5\right) \wedge\left(a_{3}=3\right) \rightarrow^{1}(D=1) \\
r_{1}^{-}: & \left(a_{1}=6\right) \wedge\left(a_{3}=8\right) \rightarrow^{1}(D \neq 1) \\
r_{2}^{-}: & \left(a_{1}=5\right) \wedge\left(a_{3}=9\right) \rightarrow^{1}(D \neq 1) \\
r_{3}^{-}: & \left(a_{1}=6\right) \wedge\left(a_{3}=3\right) \rightarrow^{1}(D \neq 1)
\end{array}
$$

Rule generalization. As indicated in the previous subsection, we can obtain decision rules directly from the reduced information system. We obtain one rule for each equivalence class of the partition $R^{*}(\mathrm{RED})$. However, rules might contain attributes whose values are irrelevant for determining the target concept. Furthermore, we can generalize rules further by inspecting which conditions in a rule can be removed without causing any inconsistency. A decision rule obtained by dropping the maximum possible number of conditions is called a maximally general rule. By construction, maximally general rules contain a minimum number of conditions. We use the decision matrix technique to find all the maximally general rules. ${ }^{10}$

Let $X_{i}^{+}, i=(1,2, \ldots \gamma)$, denote the equiva- 


\begin{tabular}{cccc}
\hline & $\boldsymbol{X}_{\mathbf{1}}^{-}$ & $\boldsymbol{X}_{\mathbf{2}}^{-}$ & $\boldsymbol{X}_{\mathbf{3}}^{-}$ \\
\hline$X_{1}{ }^{-}$ & $\left(a_{3}, 4\right)$ & $\left(a_{1}, 6\right),\left(a_{3}, 4\right)$ & $\left(a_{3}, 4\right)$ \\
$X_{2}{ }^{+}$ & $\left(a_{1}, 5\right),\left(a_{3}, 3\right)$ & $\left(a_{3}, 3\right)$ & $\left(a_{1}, 5\right)$ \\
\hline
\end{tabular}

lence classes of the relation $R^{*}(\mathrm{RED})$ such that $X_{i}^{+} \subseteq \operatorname{POS}_{\mathrm{RED}}(Y)$; and let $X_{j}^{-}, j=(1,2$, $\ldots, \rho)$, denote the equivalence classes of the relation $R^{*}(\mathrm{RED})$ such that $X_{j}^{-} \subseteq$ $\operatorname{NEG}_{\mathrm{RED}}(Y)$. We define a decision matrix $\boldsymbol{M}$ $=\left(\boldsymbol{M}_{i j}\right)_{\gamma \times \rho}$ as

$$
\begin{aligned}
& \boldsymbol{M}_{i j}=\left\{\left(a, f\left(X_{i}^{+}, a\right)\right): a \in \operatorname{RED}, f\left(X_{i}^{+}, a\right) \neq\right. \\
& \left.f\left(X_{j}^{-}, a\right)\right\}
\end{aligned}
$$

where $a$ is a condition attribute belonging to RED. That is, $\boldsymbol{M}_{i j}$ contains all attribute-value pairs whose values are not the same between the equivalence class $X_{i}^{+}$and the equivalence class $X_{\mathrm{j}}^{-}$.

We obtain the set of decision rules computed for a given equivalence class $X_{i}^{+}$by treating each element of $\boldsymbol{M}_{i j}$ as a Boolean expression and constructing the following Boolean function:

$$
B_{i}=\widehat{\wedge}_{j}\left(\vee M_{i j}\right)
$$

where $\wedge$ and $\vee$ are the usual conjunction and disjunction operators.

We can show that the prime implicants of the Boolean function $B_{i}$ are the maximally general rules for the equivalence class $X_{i}^{+}$ belonging to the positive learning region $\operatorname{POS}_{\mathrm{RED}}(Y)$. Thus, by finding the prime implicants of all the decision functions $B_{i}(i$ $=1,2, \ldots, \gamma)$, we can compute all the maximally general rules for the positive learning region $\operatorname{POS}_{\mathrm{RED}}(Y)$.

Example 5. Following from Example 4, the equivalence classes of the relation $R(\mathrm{RED})$ are

$$
\begin{aligned}
& X_{1}^{+}=X_{1}=\left\{o b j_{4}, o b j_{6}, o b j_{8}\right\} \\
& X_{2}^{+}=X_{2}=\left\{o b j_{5}\right\} \\
& X_{1}^{-}=X_{3}=\left\{o b j_{1}, o b j_{3}\right\} \\
& X_{2}^{-}=X_{4}=\left\{o b j_{2}\right\} \\
& X_{3}^{-}=X_{5}=\left\{o b j_{7}\right\}
\end{aligned}
$$

Table 4 shows the decision matrix

$$
\boldsymbol{M}=\left(\boldsymbol{M}_{i j}\right)_{2 \times 3}
$$

We then obtain the Boolean functions for each $X_{i}^{+}(i=1,2)$ as follows:

$$
\begin{aligned}
B_{1}= & \left(a_{3}=4\right) \wedge\left(\left(a_{1}=6\right) \vee\left(a_{3}=4\right)\right) \\
& \wedge\left(a_{3}=4\right)=\left(a_{3}=4\right) \\
B_{2}= & \left(\left(a_{1}=5\right) \vee\left(a_{3}=3\right)\right) \wedge\left(a_{3}=3\right) \\
& \wedge\left(a_{1}=5\right)=\left(a_{1}=5\right) \wedge\left(a_{3}=3\right)
\end{aligned}
$$

Therefore, the maximally general rule for the equivalence class $X_{1}{ }^{+}$is

$$
\left(a_{3}=4\right) \rightarrow{ }^{0.67}(D=1)
$$

and the maximally general rule for the equivalence class $X_{2}{ }^{+}$is

$$
\left(a_{1}=5\right) \wedge\left(a_{3}=3\right) \rightarrow^{1}(D=1) .
$$

Given the set of all maximally general rules for an information system $S$, our system provides the options to find the set of minimal rules and the set of minimal covering rules. The support set of a rule $r_{i}$, denoted as $\operatorname{supp}\left(r_{i}\right)$, is the collection of rows of the original table satisfying the condition part of $r_{i}$. A collection of rules RUL' is a set of minimal rules if, for every $r_{i} \in$ RUL',

$$
\operatorname{supp}\left(r_{i}\right) \nsubseteq \operatorname{supp}\left(r_{j}\right)_{\forall r_{j} \in \mathrm{RUL}^{\prime}, r_{i} \neq r_{j}}
$$

A collection of rules RUL" is a set of minimal covering rules if, for every $r_{i} \in$ RUL",

$$
\operatorname{supp}\left(r_{i}\right) \nsubseteq \bigcup \operatorname{supp}\left(r_{j}\right)_{r_{j} \in \mathrm{RUL}^{\prime \prime}, r_{i} \neq r_{j}}
$$

Example 6. As shown in Example 5, we can use a decision matrix to calculate the maximally general rule for the concept $Y_{1}$. Similarly, we can obtain the maximally general rules for concepts $Y_{0}$ and $Y_{2}$. The set of all maximally general rules for the information system in Table 2 are

$$
\begin{aligned}
& r_{1}:\left(a_{3}=8\right) \rightarrow^{1}(D=0) \\
& r_{2}:\left(a_{3}=9\right) \rightarrow^{1}(D=0) \\
& r_{3}:\left(a_{3}=4\right) \rightarrow^{0.67}(D=1) \\
& r_{4}:\left(a_{1}=5\right) \wedge\left(a_{3}=3\right) \rightarrow^{1}(D=1) \\
& r_{5}:\left(a_{1}=6\right) \wedge\left(a_{3}=3\right) \rightarrow^{1}(D=2)
\end{aligned}
$$

where $r_{1}$ and $r_{2}$ are for the concept $Y_{0}, r_{3}$ and $r_{4}$ are for the concept $Y_{1}$, and $r_{5}$ is for the concept $Y_{2}$.
The support sets of these rules are

$$
\begin{aligned}
& \operatorname{supp}\left(r_{1}\right)=\left\{o b j_{1}, o b j_{3}\right\} \\
& \operatorname{supp}\left(r_{2}\right)=\left\{o b j_{2}\right\} \\
& \operatorname{supp}\left(r_{3}\right)=\left\{o b j_{4}, o b j_{6}, o b j_{8}\right\} \\
& \operatorname{supp}\left(r_{4}\right)=\left\{o b j_{5}\right\} \\
& \operatorname{supp}\left(r_{5}\right)=\left\{o b j_{7}\right\}
\end{aligned}
$$

Because these support sets are not overlapping, the rules are both minimal rules and minimal covering rules.

\section{Experimental results}

We have implemented this method in the KDD-R (Knowledge Discovery in Databases, Rough Sets Approach) system developed at the University of Regina. ${ }^{9}$ The system performs data analysis, database mining, pattern recognition and validation, and expert-system building. We tested the proposed method by applying the KDD-R system on the data for water-demand prediction. Our objective is to analyze the set of training data and generate a set of decision rules to predict a city's daily water demand. As we mentioned, the set of training samples consists of 306 objects collected over a period of 10 months and includes information on day of the week, weather conditions, and daily water consumption.

In our experiment, we divided the values of decision attributes into nine ranges so that the information system has nine concepts. The KDD-R system generated a total of 188 rules. Table 5 lists the number of rules generated for different concepts; $N C$ stands for the number of training samples covered by the rules.

The most general rule for the concept $D=$ $(60-70]$ is

$$
\begin{aligned}
& \left(-13.80<a_{2} \leq-1.40\right) \wedge\left(81.60<a_{3} \leq\right. \\
& 93.00) \rightarrow^{1}(60<D \leq 70)
\end{aligned}
$$

This rule covers $12.37 \%$ of the training objects concluding the concept. The rule states that

If the maximum temperature is within the range $\left(-13.80^{\circ} \mathrm{C},-1.40^{\circ} \mathrm{C}\right]$ and the average humidity is within $(81.60 \%, 93 \%]$, then the water demand is between 60 megaliters and 70 megaliters, with an uncertainty factor of 1 .

The most general rule for the concept $D=$ $(70-80]$ is

$$
\begin{aligned}
& \left(a_{0}=\boldsymbol{M}\right) \wedge\left(-31.50 \leq a_{2} \leq-13.80\right) \\
& \rightarrow^{1}(70<D \leq 80)
\end{aligned}
$$


This rule covers $4.17 \%$ of the training objects concluding the concept. It states that

If the day of the week is Monday and the minimum temperature is within the range $\left[-31.50^{\circ} \mathrm{C},-13.80^{\circ} \mathrm{C}\right]$, then the water demand is between 70 megaliters and 80 megaliters, with an uncertainty factor of 1 .

The most general rule for the concept $D=$ $(80-90]$ is

$$
\begin{aligned}
& \left(a_{0}=F\right) \wedge\left(6.70<a_{1} \leq 34.10\right) \wedge(4.50 \leq \\
& \left.a_{6} \leq 8.70\right) \rightarrow^{1}(80<D \leq 90)
\end{aligned}
$$

This rule covers $7.90 \%$ of the training objects concluding the concept. It states that

If the day of the week is Friday and the maximum temperature is within the range $\left(6.70^{\circ} \mathrm{C}\right.$, $\left.34.10^{\circ} \mathrm{C}\right]$ and the average wind speed is within [4.50 km/hr., $8.70 \mathrm{~km} / \mathrm{hr}$.], then the water demand is between 80 megaliters and 90 megaliters, with an uncertainty factor of 1 .

The most general rule for the concept $D=$ $(100-110]$ is

$$
\begin{aligned}
& \left(a_{0}>S U\right) \wedge\left(47.40<a_{3} \leq 53.10\right) \wedge \\
& \left(17.10<a_{6} \leq 21.30\right) \rightarrow{ }^{1}(100<D \leq 110)
\end{aligned}
$$

This rule covers $22.22 \%$ of the training objects concluding the concept. It states that

If the day of the week is Sunday and the average humidity is within $(47.40 \%, 53.10 \%]$ and the average wind speed is within $(17.10 \mathrm{~km} / \mathrm{hr}$., $21.30 \mathrm{~km} / \mathrm{hr}$.], then the water demand is between 100 megaliters and 110 megaliters, with an uncertainty factor of 1 .

To evaluate the rules derived by our method, we conducted a leave-ten-out experiment by using $90 \%$ of the data for training and the remaining $10 \%$ for testing. The error rate depends on the selection of training samples. We conducted the experiment 10 times. The best error rate of prediction was $6.67 \%$, and the average error rate of prediction was $10.27 \%$.

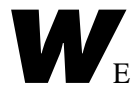

E HAVE SUGGESTED A METHOD

for generating prediction rules from a given set of training examples. The method is an extension of the rough-set model. The salient feature of the proposed method is that it uses the statistical information inherent in the

\begin{tabular}{ccr}
\hline Concepts & Number of rules & \multicolumn{1}{c}{$\boldsymbol{N C}$} \\
\hline$[53-60]$ & 3 & 3 \\
$(60-70]$ & 43 & 97 \\
$(70-80]$ & 67 & 120 \\
$(80-90]$ & 32 & 38 \\
$(90-100]$ & 16 & 18 \\
$(100-110]$ & 8 & 9 \\
$(110-120]$ & 6 & 7 \\
$(120-130]$ & 4 & 5 \\
$(130-140]$ & 3 & 3 \\
$(140-176]$ & 5 & 6 \\
\hline
\end{tabular}

knowledge system. Thus, our method can derive decision rules from incomplete knowledge. This capability is important, because we seldom have complete and consistent information when designing intelligent systems.

Application of this knowledge-discovery method for water-demand prediction complements manual knowledge-acquisition techniques (see the sidebar, "The intelligent system"). It offers the advantage of describing important relationships between condition factors and water consumption in terms of simple if-then rules that users can easily understand. The experimental results indicate that the proposed algorithm can generate rules for water-demand prediction, providing more precise information than is available through knowledge acquisition from human experts.

Our proposed method for machine induction is general and can be applied to other domains, such as general consumer-demand prediction, fault diagnosis, and process control. For water-demand prediction, we have also tried different sets of causal variables with the rule-induction method to further improve predictive accuracy. The results show that adding time-series data, such as yesterday's and the day before yesterday's water consumption, as conditional variables gives better results than using weather factors alone. In terms of the

\section{The intelligent system}

The knowledge-discovery module for water-demand prediction is one component of the intelligent system for monitoring and controlling the water-distribution system. The intelligent system consists of five modules:

- the expert-system module,

- the energy-management module,

- the water-demand prediction module,

- the pipeline-network-simulation module, and

- the scheduling and planning module.

The expert system consists of a knowledge base for detecting faults and recommending a series of adjustments on equipment at a given state of operations.

The energy-management module informs users of the most cost-effective combination of pumps and valves among the possible configurations suggested by the expert system.

The prediction module forecasts water demands. It has been implemented using various AI techniques, including neural networks, fuzzy sets, case-based reasoning, and knowledge discovery from databases.

The work presented in the main text of the article describes the knowledge-discovery component of the prediction module. The forecast on water usage, along with the recommendations on pumps and valve adjustments provided by the expert system, becomes input to the simulation module, for verification purposes.

The simulation program uses an extended period simulation to represent the dynamic behavior of flows, pressures, and water levels. The operational procedure of pumps and valves suggested by the expert system are satisfactory if the simulation module shows that

- the pressure at the outlet of each pumping station is within the set-point limit,

- the water level remains within the range allowable for the reservoirs, and

- the combination of pumps and valves does not cause any reservoir to overflow or deplete within a short period of time.

The project began in May 1994, and we have completed the prototype of the expert system the simulation program, the prediction module, and the energy-management module. We are working on the scheduling and planning module, and on integrating the different components into an intelligent system. We expect to complete the project this fall. 
machine-induction method, we have developed a new algorithm that integrates rule induction with case-based reasoning to improve case retrieval and problem solving. We have applied this integrated method to numeric-prediction domains. Experimental results show that case-based reasoning with rule induction performs better than casebased reasoning by itself, in terms of predictive accuracy.

\section{Acknowledgments}

This research is supported by the Telecommunications Research Laboratories and the Natural Science and Engineering Research Council of Canada. We deeply appreciate the cooperation of the personnel at the City of Regina's Municipal Water Engineering and Water Supply departments. We also would like to thank Paitoon Tontiwachwuthikul for his invaluable suggestions on this work.

\section{References}

1. J. Quevedo et al., "Time Series Modelling of Water Demand: A Study on Short-Term and Long-Term Predictions," in Computer Applications in Water Supply, Vol. 1, B. Coulbeck and C. Orr, eds., Research Studies Press Ltd. Letchworth, England, 1988, pp. 268-288.

2. R.S. Michalski, J.G. Carbonell, and T.M Mitchell, eds., Machine Learning: An Artificial Intelligence Approach, Vol. 1, Morgan Kaufmann, San Francisco, 1983.

3. R.S. Michalski, J.G. Carbonell, and T.M. Mitchell, eds., Machine Learning: An Artificial Intelligence Approach, Vol. 2, Morgan Kaufmann, 1986.

4. Z. Pawlak, Rough Sets: Theoretical Aspects of Reasoning about Data, Kluwer Academic Publishers, Norwell, Mass., 1991.

5. J.R. Quinlan, "Induction of Decision Trees," Machine Learning, Vol. 1, No. 1, 1986, pp. 81-106.

6. W. Ziarko, "Variable Precision Rough Set Model," J. Computer and System Sciences, Vol. 46, No. 1, 1993, pp. 39-59.
7. J. Katzberg and W. Ziarko, "Variable Precision Rough Sets with Asymmetric Bounds," in Rough Sets, Fuzzy Sets and Knowledge Discovery, W. Ziarko, ed., Springer-Verlag, New York, 1994, pp. 167-177.

8. Z. Pawlak, S.K.M. Wong, and W. Ziarko, "Rough Sets: Probabilistic Versus Deterministic Approach," Int'l J. Man-Machine Studies, Vol. 29, No. 1, 1988, pp. 81-95.

9. W. Ziarko and N. Shan, "KDD-R: A Comprehensive System for Knowledge Discovery in Databases Using Rough Sets," Proc. Third Int'l Workshop Rough Sets and Soft Computing, San Jose State Univ., San Jose, Calif. 1994, pp. 164-173.

10. W. Ziarko and N. Shan, "A Rough Set-Based Method for Computing All Minimal Deterministic Rules in Attribute-Value Systems,' Computational Intelligence: An Int'l J., Vol. 12, No. 2, 1996, pp. 223-234.

11. S.K.M. Wong and W. Ziarko, "INFER-An Adoptive Decision Support System Based on the Probabilistic Approximate Classification,' Proc. Sixth Int'l Workshop Expert Systems and Their Applications, Agence de Informatique, Avignon, France, 1986, pp. 713-726.

Aijun An is a PhD candidate in the Department of Computer Science at the University of Regina. Her research interests include machine learning, casebased reasoning, knowledge engineering for developing real-time expert systems, validation and verification of knowledge-based systems, and applications of artificial intelligence techniques to practical problems. She received a BSc and an MSc in computer science from Xidian University, $\mathrm{Xi}$ 'an, China. She is a student member of the AAAI and the IEEE Computer Society. Readers can contact An at the Dept. of Computer Science, Univ. of Regina, Regina, SK S4S 0A2, Canada; aijun@cs.uregina.ca.

Christine Chan is an associate professor in the Department of Computer Science at the University of Regina. Her research interests include industrial applications of artificial intelligence, object-oriented methodologies for knowledgebased systems development, knowledge acquisition and conceptual modeling, and educational instructional software. She received MSc degrees in computer science and management information systems from the University of British Columbia. She also received a $\mathrm{PhD}$ in the interdisciplinary studies of computer science, philosophy, and psychology from Simon Fraser University. She is a member of the AAAI, the IEEE Computer Society, the ACM, and the Canadian
Information Processing Society. Readers can contact Chan at the Dept. of Computer Science, Univ. of Regina, Regina, SK S4S 0A2, Canada; chan@ cs.uregina.ca; http://www.cs.uregina.ca/ chan.

Ning Shan is a PhD candidate in the Department of Computer Science at the University of Regina. His research interests include machine learning, rough sets, rule-based expert systems, knowledge discovery in databases, and real-world applications of artificial intelligence. He received a B.Eng. in computer engineering from ChengDu Electronic Engineering Institute, China, and an MSc in computer science from the University of Regina. Readers can contact Shan at the Dept. of Computer Science, Univ. of Regina, Regina, SK S4S 0A2, Canada; ning@cs.uregina.ca.

Nick Cercone is a professor and the chair of the Computer Science Department at the University of Waterloo. His research interests include naturallanguage processing, knowledge-based systems, knowledge discovery in databases, and design and human interfaces. He received a BS in engineering science from the University of Steubenville, an MS in computer and information science from Ohio State University, and a $\mathrm{PhD}$ in computing science from the University of Alberta. He is a member of the ACM, the IEEE, the AAAI, the Artificial Intelligence Simulation of Behavior, the AGS, and the Association of Computational Linguistics. He recently won the Canadian Artificial Intelligence Society's Distinguished Service Award. Readers can contact Cercone at the Computer Science Dept., Faculty of Mathematics, Univ. of Waterloo, Waterloo, ON N2L 3G1, Canada; ncercone@uwaterloo.ca.

Wojciech Ziarko is a professor in the Compute Science Department at the University of Regina. His research interests are knowledge discovery in databases, machine learning, pattern classification, and control-algorithm acquisition from sensor data. These research interests are, to a large degree, motivated by the recent introduction of the theory of rough sets, which serves as a basic mathematical framework in much of his work. He received an MSc in applied mathematics from Warsaw University of Technology, Poland, and a PhD in computer science, specializing in databases, from the Institute of Computer Science of the Polish Academy of Sciences, Warsaw, Poland. Readers can contact Ziarko at the Dept. of Computer Science, Univ. of Regina, Regina, SK S4S 0A2, Canada; ziarko@cs.uregina.ca. 